\title{
Duration of diabetes, glucose control and cardiovascular risk
}

\author{
N. V. Emanuele
}

Received: 10 September 2009 / Accepted: 22 September 2009 /Published online: 20 October 2009

(C) Springer-Verlag 2009

Keywords Cardiovascular risk · Intensive glucose control · Type 2 diabetes mellitus

$\begin{array}{ll}\begin{array}{l}\text { Abbreviations } \\ \text { ACCORD }\end{array} & \begin{array}{l}\text { Action to Control Cardiovascular Risk in } \\ \text { Diabetes } \\ \text { Action in Diabetes and Vascular Disease: } \\ \text { Preterax and Diamicron Modified Release }\end{array} \\ & \begin{array}{l}\text { Controlled Evaluation } \\ \text { Collaborators on Trials of Lowering Glucose }\end{array} \\ \text { CONTROL } & \begin{array}{l}\text { United Kingdom Prospective Diabetes Study } \\ \text { UKPDS }\end{array} \\ \text { VADT } & \text { Veterans Affairs Diabetes Trial }\end{array}$

To the Editor: We read with great interest the meta-analysis by the Collaborators on Trials of Lowering Glucose (CONTROL) Group of recent trials examining the impact of intensive glycaemic control on cardiovascular risk in people with type 2 diabetes mellitus [1]. This well executed study analysed data on 27,049 patients in four important recently published prospective trials, the Action to Control Cardiovascular Risk in Diabetes (ACCORD) study [2], the Action in Diabetes and Vascular Disease: Preterax and Diamicron Modified Release Controlled Evaluation (ADVANCE) study [3], the Veterans Affairs Diabetes Trial (VADT) [4] and the United Kingdom Prospective Diabetes Study (UKPDS) [5].

The main conclusion was that the primary composite outcome, death from cardiovascular causes including sudden death, non-fatal myocardial infarction and non-fatal stroke,

\section{N. V. Emanuele $(\triangle)$}

Loyola University Medical Center,

2160 S. First Avenue, Fahey Center (Building 54), Room 137,

Maywood, IL 60153, USA

e-mail: nemanue@lumc.edu was reduced by $9 \%$ (HR $0.91,95 \%$ CI $0.84-0.99)$ in those individuals allocated to more intensive glycaemic control compared with those with less intensive control over an average follow-up time of 4.4 years [1]. There was no evidence of heterogeneity among the trials. This result was driven largely by a $15 \%$ reduction in myocardial infarction in more intensively treated patients, with no beneficial effects seen on stroke, heart failure, all-cause mortality or cardiovascular mortality. The effect was consistent across most prespecified subgroups (sex, age, $\mathrm{HbA}_{1 \mathrm{c}}$, duration of diabetes and history of microvascular disease), but there was one exception. Patients who had not had a macrovascular event before randomisation had cardiovascular benefit from tight glycaemic control (HR 0.84, 95\%CI 0.75-0.94), whereas those who had a macrovascular event before randomisation had no cardiovascular benefit from tight glycaemic control (HR 1.00, 95\% CI 0.89-1.13).

The meta-analysis showed that this benefit was not achieved without a price. First, those randomised to more intensive blood glucose-lowering suffered a more than doubling of the risk for severe hypoglycaemia compared with the less intensive patients (HR 2.48, 95\% CI 1.91-3.21). Second, there were small trends suggesting increased allcause mortality (HR 1.04, 95\% CI 0.90-1.20) and especially increased cardiovascular mortality (HR 1.10, 95\% CI 0.84 1.42) in those individuals who were treated to lower glycaemic targets when all the studies were analysed together. This $10 \%$ trend for a relative increase in cardiovascular death showed significant heterogeneity among the four studies and led the CONTROL Group to acknowledge appropriately that '... the possibility of harm with more-intensive glycaemic treatment cannot be ruled out'. [1].

Despite the appropriate caution sounded by the CONTROL Group, we are deeply concerned with the message that this meta-analysis might send and felt it would be worthwhile to suggest a slightly different appraisal of the 
Table 1 Intensive glycaemic control and clinical events hazard ratios

\begin{tabular}{llllll}
\hline Event & \multicolumn{2}{l}{ Earlier diabetes } & & \multicolumn{2}{l}{ More advanced diabetes } \\
\cline { 2 - 3 } \cline { 5 - 6 } & UKPDS & ADVANCE & & ACCORD & VADT \\
\hline CV mortality & 1.02 & 0.88 & & 1.35 & 1.32 \\
All cause mortality & 0.96 & 0.93 & & 1.22 & 1.07 \\
\hline
\end{tabular}

data. It seems to us that, clinically, there are two distinctly different populations involved in the four trials and that mixing these two populations may obscure the important finding that good glycaemic control may produce cardiovascular benefit early, but not late in the evolution of the disease.

The populations in the VADT and the ACCORD study were advanced in the progression of their diabetes. The median duration of diabetes was a decade in both studies. $\mathrm{HbA}_{1 \mathrm{c}}$ values were high (ACCORD 8.1\%, VADT 9.4\%), despite the fact that $35 \%$ (ACCORD) to $50 \%$ (VADT) of the individuals were on insulin at entry. At the other end of the spectrum were the patients in UKPDS, with newly diagnosed diabetes and relatively good $\mathrm{HbA}_{1 \mathrm{c}}$ levels $(7.1 \%)$ without medication. In the middle were patients in the ADVANCE study. Though the median duration of diabetes was 7 years at entry, not markedly different from the VADT and the ACCORD study, other characteristics suggest that their disease process was clinically and biologically much less advanced than those in the VADT and the ACCORD study. Specifically, the mean entry $\mathrm{HbA}_{1 \mathrm{c}}$ level of the ADVANCE study participants was $7.5 \%$, with only about $1.5 \%$ taking insulin, very distinctly different from the values in the VADT and the ACCORD study. When the four studies are viewed as two populations (very to relatively early diabetes in the UKPDS and the ADVANCE study and later diabetes in the VADT and the ACCORD study), rather than as one population, as was done in the meta-analysis, an important difference becomes very clear.

While there were consistent, statistically insignificant trends toward benefit in the primary endpoint in each of the studies that became significant when the data from all four studies was combined, the same happy result was not seen in two extremely important secondary endpoints: cardiovascular mortality and all-cause mortality (Table 1). In the ACCORD study, there was a statistically significant $35 \%$ increase in cardiovascular mortality and $22 \%$ increase in all-cause mortality with better glycaemic control. Indeed, the glycaemic arm of the ACCORD study was stopped 1 year early because of these findings. The VADT did not report any statistically significant detriment from intensive control (other than increased rates of hypoglycaemia). However, examination of trends shows similar findings as in the ACCORD study. With more intensive glycaemic control, there was a $32 \%$ increase in cardiovascular mortality and a $7 \%$ increase in all-cause mortality in the VADT, both in the same direction as in the ACCORD study. Neither was statistically significant, presumably because of the smaller size of the VADT population and the smaller number of events. So, in reality, the effects of meticulous control were in the same direction in the ACCORD study and the VADT. That is, there was detriment in terms of CV and all-cause mortality in both trials that randomised people with more advanced diabetes, though it was statistically significant only in the ACCORD study. In contrast, the trends for cardiovascular and all-cause mortality were in a beneficial direction or essentially neutral in the other two studies, which enrolled people with less advanced diabetes. Cardiovascular death was reduced by $12 \%$ (not significant) in the ADVANCE study and increased by only $2 \%$ (not significant) in the UKPDS. There were insignificant trends towards decreased all-cause mortality by $7 \%$ in the ADVANCE study and by $4 \%$ in the UKPDS.

In summary the CONTROL Group are to be congratulated on their excellent meta-analysis. However, although our view is less statistically rigorous, we think it prudent and clinically sensible to consider VADT and the ACCORD study vs the ADVANCE study and the UKPDS as two separate populations: people with later vs earlier diabetes with different cardiovascular responses to intensification of glycaemic control.

Duality of interest The author declares that there is no duality of interest associated with this manuscript.

\section{References}

1. Turnbull FM, Abraira C, Anderson RJ et al (2009) Intensive glucose control and macrovascular outcomes in type 2 diabetes. Diabetologia 52:2288-2298 Erratum 52:2470

2. The Action to Control Cardiovascular Risk in Diabetes Study Group (2008) Effects of intensive glucose lowering in type 2 diabetes. N Engl J Med 358:2545-2559

3. The ADVANCE Collaborative Group (2008) Intensive blood glucose control and vascular outcomes in patients with type 2 diabetes. N Engl J Med 358:2560-2572

4. Duckworth W, Abraira C, Moritz T et al (2009) Glucose control and vascular complications in veterans with type 2 diabetes. N Engl J Med 360:129-139

5. UK Prospective Diabetes Study (UKPDS) Group (1998) Intensive blood-glucose control with sulphonylureas or insulin compared with conventional treatment and risk of complications in patients with type 2 diabetes (UKPDS 33). Lancet 352:837-853 